\title{
Efeito leishmanicida in vitro de Stachytarpheta cayennensis (Rich.) Vahl (Verbenaceae)
}

\author{
Rosilene C.R. Moreira', Graciomar C. Costa', Thaiana C. Lopes ${ }^{2}$, Jeamile L. Bezerra ${ }^{3}$, Rosane \\ N.M. Guerra' ${ }^{2}$ José Manuel M. Rebêlo ${ }^{4}$, Maria Nilce S. Ribeiro ${ }^{3}$, Flávia R.F. Nascimento ${ }^{2}$, \\ Jackson M.L. Costa ${ }^{5 *}$
}

\author{
${ }^{1}$ Centro Universitário do Maranhão - UNICEUMA, Rua Josué Montello, $n^{\circ} 1$, Renascença II, 65075-120, \\ São Luís, MA, Brasil, \\ ${ }^{2}$ Laboratório de Imunofisiologia, Departamento de Patologia, Universidade Federal do Maranhão, \\ Campus do Bacanga, Av. dos Portugueses s/n, 65085-580, São Luís, MA, Brasil, \\ ${ }^{3}$ Laboratório de Farmacognosia, Departamento de Farmácia, Universidade Federal do Maranhão, \\ Campus do Bacanga, Av. dos Portugueses s/n, , 65085-580, São Luís, MA, Brasil, \\ ${ }^{4}$ Laboratório de Entomologia e Vetores, Departamento de Biologia, Praça da Madre Deus, $n^{\circ} 2$, \\ 65025-580, São Luís, MA, Brasil, \\ ${ }^{5}$ Centro de Pesquisas Gonçalo Moniz, Fiocruz-BA, Rua Waldemar Falcão, $n^{\circ} 121$, Brotas, \\ 40295-001, Salvador, BA, Brasil
}

\begin{abstract}
RESUMO: A atividade anti-Leishmania do extrato hidroalcoólico de Stachytarpheta cayennensis, espécie utilizada popularmente no tratamento de lesões cutâneas causadas por Leishmania sp, foi testado em ensaios in vitro utilizando formas promastigotas de Leishmania braziliensis e L. amazonensis. O extrato hidroalcoólico foi preparado a partir das folhas secas e utilizado em culturas de L. amazonensis e L. braziliensis nas concentrações de 500 a $32,5 \mu \mathrm{g} / \mathrm{mL}$. Após 24 horas as formas promastigotas foram quantificadas para o cálculo da $\mathrm{CI}_{50}$. A citotoxicidade do extrato foi avaliada também em culturas de macrófagos peritoneais. O extrato apresentou efeito leishmanicida dose e espécie-dependente para promastigotas de Leishmania sendo mais eficaz para L. braziliensis. $\mathrm{O}$ extrato não apresentou efeito citotóxico quando utilizado nas culturas de macrófagos. Concluiuse que o extrato hidroalcoólico de S. cayennensis inibe formas promastigotas de Leishmania in vitro o que poderia justificar, pelo menos parcialmente, o uso popular dessa espécie no tratamento de úlceras causadas por Leishmania.
\end{abstract}

Unitermos: Starchytarpheta cayennensis, Verbenaceae, Leishmania braziliensis, Leishmania amazonensis.

\begin{abstract}
In vitro leishmanicidal effect of Stachytarpheta cayennensis (Rich.) Vahl (Verbenaceae)". Leishmanicidal activity of the hydroalcoholic extract of Stachytarpheta cayennensis, species that is usually employed in ulcers caused by Leishmania, was evaluated in vitro using Leishmania braziliensis and L. amazonensis promastigotes forms. The hydroalcoholic extract was prepared from dried leaves and used in L. amazonensis and L. braziliensis promastigotes cultures at concentrations of 500 to $32.5 \mu \mathrm{g} / \mathrm{mL}$. After 24 hours the promastigotes forms were quantified and the $\mathrm{IC}_{50}$ was calculated. The cytotoxicity of the extract was evaluated using peritoneal macrophages. The extract presented a dose and specie-dependent leishmanicidal effect to Leishmania promastigotes, mainly to the L. braziliensis ones. The cytotoxic effect was not observed in macrophage cultures. In conclusion, the hydroalcoholic extract of $S$. cayennensis inhibits the growing of Leishmania promastigotes forms in vitro accounting for the folk use of this vegetal in skin ulcers caused by Leishmania.
\end{abstract}

Keywords: Starchytarpheta cayennensis, Verbenaceae, Leishmania braziliensis, Leishmania amazonensis.

\section{INTRODUÇ̃̃O}

As leishmanioses são doenças endêmicas causadas por protozoários do gênero Leishmania ocorrendo em várias partes do mundo, ocasionando um elevado índice de morbidade e mortalidade. Seu tratamento é feito à base de antimoniais pentavalentes
$\left(\mathrm{Sb}^{+5}\right)$, anfotericina $\mathrm{B}$ e pentamidinas (isotionato e mesilato), quimioterápicos de alto custo, usados por via parenteral, requerendo um período de administração prolongado, causando efeitos adversos, como: alterações cardíacas, renais, pancreáticas e hepáticas (Olliaro; Bryceson, 1997; Croft; Yardley, 2002).

Pouco tem sido feito desde a introdução dos $\mathrm{Sb}^{+5}$ 
na pesquisa de novos quimioterápicos para o tratamento das leishmanioses. Entretanto, as dificuldades em relação à terapêutica atual têm conduzido a estudos com o objetivo de avaliar terapias alternativas, como a fitoterapia (Phillipson; Wright, 1991a,b). As plantas são importantes fontes para a investigação de produtos biologicamente ativos. Cerca de um quarto dos medicamentos do mercado farmacêutico possuem extratos em sua composição, alguns dos quais têm sido, inclusive, utilizados como matéria-prima de drogas semi-sintéticas (Bergmann et al., 1997).

O uso de plantas no tratamento da Leishmaniose Tegumentar Americana (LTA) é uma prática antiga entre as populações das áreas endêmicas (Fournet et al., 1992a; Moreira et al., 1998; 2002). Geralmente, as preparações vegetais são utilizadas por via oral ou tópica sobre as lesões cutâneas (Netto et al., 1985; Iwu et al., 1994; Akendengue et al., 1999). Porém, esses tratamentos são empíricos e pouco se sabe sobre sua real eficácia, uma vez que na LTA pode ocorrer a cura espontânea das lesões (Marsden et al., 1984; Costa et al., 1990; Rossel et al., 1992; Marsden, 1994).

Estudo realizado por nosso grupo no Município de Buriticupu (Amazônia do Maranhão), Brasil, área endêmica para LTA, demonstrou que dentre as preparações vegetais utilizadas pela população no tratamento das úlceras causadas por Leishmania sp, uma das mais citadas foi o extrato de folhas de Stachytarpheta cayennensis (Moreira et al., 2002).

Stachytarpheta cayennensis (Rich.) Vahl, conhecida popularmente como gervão, rinchão e vassourinha-de-botão pertence à família Verbenaceae (Pio Correa, 1984). Esta espécie é encontrada na América tropical e subtropical, do México até o Brasil (Lopes, 1977; Troncoso, 1979) e tem sido utilizada na medicina tradicional como anti-inflamatória, analgésica, antipirética, hepatoprotetora, laxante e no tratamento de distúrbios gástricos (Mathias; Emily, 1993; Mesia-Vela et al., 2004). A aplicação de folhas e raízes trituradas também é usada no tratamento de lesões de pele (Caribe; Campos, 1991) inclusive, em lesões ulceradas causadas por Leishmania sp (Moreira et al, 2002).

Alguns dos efeitos preconizados pela população já foram demonstrados experimentalmente, como a atividade anti-inflamatória, analgésica, gastroprotetora e antimicrobiana (Schapoval et al., 1998; Mesia-Vela et al., 2004; Duarte et al., 2004; Falcão et al., 2005). Em sua composição química apresenta, alcalóides, glicosídios (verbenalina e verbenina), taninos, saponinas, flavonóides, esteróides, quinonas, compostos fenólicos e ácido glicogênico, sendo que alguns destes constituintes químicos também estão contidos em várias espécies vegetais com efeitos leishmanicidas (Mathias; Emily, 1993).

Baseado no uso popular e nos constituintes químicos da $S$. cayennensis que indicam um potencial efeito anti-Leishmania, este estudo tem por objetivo testar o efeito do extrato hidroalcoólico das folhas de $S$. cayennensis sobre formas promastigotas de Leishmania braziliensis e L. amazonensis, principais agentes causadores da LTA.

\section{MATERIAL E MÉTODOS}

\section{Material vegetal}

As folhas de Stachytarpheta cayennensis (gervão) foram coletadas em fevereiro de 2001 no Município de Buriticupu (Amazônia), Maranhão, Brasil. A identificação foi realizada no laboratório de Botânica da Universidade Federal do Maranhão - UFMA. A exsicata encontra-se depositada no Herbário Ático Seabra da UFMA sob o $\mathrm{N}^{\mathrm{o}} 01081$.

\section{Preparação do extrato hidroalcoólico}

As folhas secas e trituradas de $S$. cayennensis ( $250 \mathrm{~g}$ ) foram extraídas por maceração com etanol a $70 \%$. A mistura permaneceu sob agitação durante seis dias, sendo em seguida submetida à filtração e concentração em rotoevaporador à pressão reduzida. Os resíduos obtidos foram então diluídos em solução tamponada de fosfato (PBS pH 7,2) a uma concentração de $5 \mathrm{mg} / \mathrm{mL}$ e foram estocados a $-20^{\circ} \mathrm{C}$ até o momento do uso.

\section{Atividade leishmanicida in vitro}

A atividade leishmanicida dos extratos foi avaliada pela inibição do crescimento de formas promastigotas de L. amazonensis (MHOM/BR/90/ BA 125) e L. braziliensis (MHOM/BR/94/H-3227) gentilmente cedidas por Dra. Aldina Barral do Centro de Pesquisas Gonçalo Moniz-CPqGM/FIOCRUZBA. As formas promastigotas foram cultivadas em meio Schneider (Sigma, Chemical Co, ST Louis, USA) suplementado com soro fetal bovino inativado (Sigma), urina humana estéril, L-glutamina $2 \mathrm{mM}$ (Gibco BRL, Grand Island, NY) e antibióticos [100 U/mL penicilina, $100 \mu \mathrm{g} / \mathrm{mL}$ de streptomicina (Sigma)]. Antes de cada experimento observou-se ao microscópio a motilidade flagelar dos parasitos. As promastigotas foram lavadas em salina estéril, contadas em câmara de Neubauer e ajustadas para a concentração de $5 \times 10^{6} / \mathrm{mL}$.

Para os ensaios, o extrato previamente obtido foi diluído cinco vezes em meio Schneider completo, obtendo-se a concentração de $1 \mathrm{mg} / \mathrm{mL}$. Deste, foram retirados $100 \mu \mathrm{L}$ os quais foram diluídos seriadamente, em placas de 96 poços de fundo chato (Costar), na proporção de 1:2 em meio Schneider completo, resultando nas concentrações finais de 500, 250, 125, 62,5 e 31,25 $\mu \mathrm{g} / \mathrm{mL}$. Em cada poço, foram então adicionados 10 $\mu \mathrm{L}$ de uma suspensão de formas promastigotas de $L$. amazonensis ou L. braziliensis contendo $5 \times 10^{6}$ células/ $\mathrm{mL}$. As mesmas concentrações foram utilizadas com o 
Tabela 1. Percentual de morte de macrófagos e promastigotas de L. braziliensis e L. amazonensis cultivados por 24 horas em presença do extrato hidroalcoólico de Stachytarpheta cayennensis e Glucantime ${ }^{\circledR}$.

\begin{tabular}{lcccc}
\hline & \multicolumn{2}{c}{ S. cayennensis } & \multicolumn{2}{c}{ Glucantime ${ }^{\circledR}$} \\
\cline { 2 - 5 } & $250 \mu \mathrm{g} / \mathrm{mL}$ & $500 \mu \mathrm{g} / \mathrm{mL}$ & $250 \mu \mathrm{g} / \mathrm{mL}$ & $500 \mu \mathrm{g} / \mathrm{mL}$ \\
\hline L. braziliensis & $74 \%$ & $100 \%$ & $56 \%$ & $100 \%$ \\
L. amazonensis & $60 \%$ & $63 \%$ & $27 \%$ & $56 \%$ \\
Macrófagos & $0 \%$ & $23 \%$ & $0 \%$ & $0 \%$ \\
\hline
\end{tabular}

Tabela 2. Avaliação da $\mathrm{CI}_{50}$ do extrato hidroalcólico de folhas de Stachytarpheta cayennensis em culturas de L. brasiliensis, L. amazonensis e macrófagos.

\begin{tabular}{lcc}
\hline Alvo & S. cayennensis & Glucantime ${ }^{\circledR}$ \\
\hline L. braziliensis & $73,7^{\mathrm{a}}$ & 98,2 \\
L. amazonensis & 382,5 & 440,3 \\
Macrófagos & 1441,4 & --- \\
\hline
\end{tabular}

Glucantime ${ }^{\circledR}$ que foi utilizado como droga de referência. O controle negativo foi obtido a partir de cultura das formas promastigotas, em meio Schneider completo.

A atividade leishmanicida dos extratos foi avaliada pela inibição do crescimento de formas promastigotas após $24 \mathrm{~h}$ de incubação a $26{ }^{\circ} \mathrm{C}$, pela contagem do número total de promastigotas vivas, levando-se em consideração a motilidade flagelar, utilizando-se câmara de Neubauer e microscópio ótico de luz comum. A contagem foi comparada com o controle do crescimento das formas promastigotas sem os extratos. Cada concentração dos extratos foi analisada em triplicata. Os resultados foram expressos como concentração inibitória do crescimento parasitário $\left(\mathrm{CI}_{50}\right)$, baseado em Schmeda-Hirschman et al. (1996) e Camacho et al. (2003).

\section{Atividade citotóxica in vitro}

Camundongos da linhagem Balb/c foram injetados com $1 \mathrm{~mL}$ de tioglicolato $3 \%$ e após 3 dias tiveram suas cavidades peritoneais lavadas com $5 \mathrm{~mL}$ de meio RPMI (Sigma) contendo heparina (10 U/mL) para obtenção das células peritoneais. Estas células continham mais que $95 \%$ de macrófagos. As células foram centrifugadas $\left(1200 \mathrm{rpm}, 4{ }^{\circ} \mathrm{C}\right)$, lavadas com salina a $0,85 \%$ e ajustadas para a concentração de $2 \mathrm{x}$ $10^{6}$ macrófagos $/ \mathrm{mL}$ de meio RPMI sem fenol vermelho acrescido de 5\% soro fetal bovino, L-glutamina e antibióticos.

Para o ensaio de citotoxidade foi utilizado o método de MTT de acordo com o descrito por Mosmann (1983). Brevemente, $2 \times 10^{5}$ macrófagos $/ 100 \mu \mathrm{L}$ foram cultivados em placas de 96 poços, fundo chato, na presença do extrato de $S$. cayennensis nas concentrações de $500,250,125,62,5$ e $31,25 \mu \mathrm{g} / \mathrm{mL}$. Em alguns poços os macrófagos foram cultivados apenas em meio RPMI completo, sem a presença do extrato, como controle.
As culturas foram incubadas por 24 horas a $37{ }^{\circ} \mathrm{C}$ em atmosfera úmida contendo $5 \%$ de $\mathrm{CO}_{2}$. Após esse período, o sobrenadante foi colhido e as placas lavadas cuidadosamente com PBS para retirar todo o extrato. Foram adicionados a cada poço $100 \mu \mathrm{L}$ de meio RPMI completo e ainda $10 \mu \mathrm{L}$ de MTT (Sigma). Após 3 horas de incubação a $37^{\circ} \mathrm{C}$ em estufa úmida e ao abrigo da luz, foram adicionados em cada poço $100 \mu \mathrm{L}$ de uma solução de SDS (Sigma) 10\%/HCL. As placas foram incubadas em temperatura ambiente "overnight" e foram então lidas em leitor de microplacas utilizando filtro de $540 \mathrm{~nm}$. A cictoxicidade foi calculada com base na seguinte fórmula: $\% \mathrm{c}=100-(\mathrm{DO} \mathrm{Mo}+$ extrato x $100 / \mathrm{DO} \mathrm{Mo}+$ meio $)$.

\section{Análise estatística}

Todos os ensaios foram realizados em triplicata e repetidos pelo menos uma vez, sendo expressos como concentração inibitória do crescimento parasitário $\left(\mathrm{CI}_{50}\right)$ calculado por regressão linear. Os dados foram comparados por teste $t$ de Student, sendo o nível de significância de $p \leq 0,05$.

\section{RESULTADOS}

O efeito anti-Leishmania do extrato hidroalcoólico de Stachytarpheta cayennensis foi mais evidente para a espécie $L$. braziliensis que para $L$. amazonensis. A maior concentração testada $(500 \mu \mathrm{g} / \mathrm{mL})$ matou $100 \%$ das formas promastigotas de L. braziliensis, porém, apresentou apenas $63 \%$ de citotoxicidade contra L. amazonensis (Tabela 1). Esse efeito foi portanto, espécie-dependente e restrito para Leishmania pois houve apenas $23 \%$ de morte de macrófagos nessa mesma concentração.

Esta diferença se refletiu também na diferença da $\mathrm{CI}_{50}$, uma vez que foi observado que a concentração do extrato necessária para matar $50 \%$ das formas

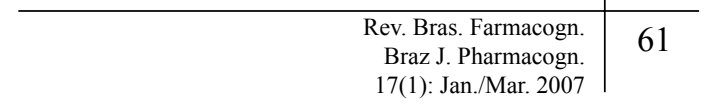


promastigotas de L. braziliensis foi de $73,7 \mu \mathrm{g} / \mathrm{mL}$, enquanto a requerida para matar L. amazonensis foi de $382,5 \mu \mathrm{g} / \mathrm{mL}$ (Tabela 2).

\section{DISCUSSÃO}

Recentes estudos demonstraram o uso de plantas medicinais no tratamento de lesões ulceradas da leishmaniose tegumentar e também em modelos in vitro (França et al., 1993; Fournet et al., 1992b; Akendengue et al., 1999; Moreira et al., 2002; Mesia-Vela et al., 2004; Paula-Junior et al., 2006; Bezerra et al., 2006).

Compostos químicos, isolados de extratos vegetais, demonstraram eficácia comprovada na atividade leishmanicida in vitro sobre formas promastigotas e/ ou amastigotas de Leishmania. Dentre eles, podem ser citados os terpenóides (Yang; Liew, 1992; Camacho et al., 2000), aminoglicosteróides e aminosteróides (Kam et al., 1997), naftoquinonas (Fournet et al., 1992a; Kayser et al., 2000), chalconas (Chen et al., 1993), glicosídios iridóides (Mittal et al., 1998); flavonóides (Araújo et al., 1998), neolignanas (Barata et al., 2000) e os alcalóides (Mahiou et al., 1994; Fournet et al., 1994; 1996; Queiroz et al., 1996; Akendengue et al., 1999).

Apesar dos estudos nesta área, ainda existem muitas espécies vegetais com potencial atividade leishmanicida a serem avaliadas. Uma dessas espécies é S. cayennensis cujas folhas têm sido usadas no tratamento da lesão ulcerada da leishmaniose tegumentar (Moreira et al., 2002), possuindo dentre os seus constituintes químicos alcalóides, glicosídios (verbenalina e verbenina) e flavonóides, substâncias com efeito leishmanicida comprovado (Mathias; Emily, 1993).

Neste estudo nós demonstramos que a utilização de extrato hidroalcoólico de folhas secas de S. cayennensis induziu a morte das formas promastigotas in vitro. Este efeito foi espécie-dependente, sendo mais evidente na espécie L. braziliensis do que em L. amazonensis e não foi decorrente de um efeito citotóxico inespecífico, uma vez que o extrato não alterou signficativamente a viabilidade dos macrófagos. Pode-se supor que este efeito diferencial esteja relacionado ao fato de que as formas promastigotas de L. braziliensis sejam mais susceptíveis em cultura que L. amazonensis, isto explicaria inclusive o fato do Glucantime ${ }^{\circledR}$ também ter tido maior eficácia em L. braziliensis do que em L. amazonensis. Vale ressaltar, que não houve diferenças no suporte nutricional do meio de cultura utilizado nas duas espécies, sendo o efeito diferencial associado com o próprio metabolismo da $L$. braziliensis.

Em concentrações acima de $1000 \mu \mathrm{g} / \mathrm{mL}$ o extrato inibiu o crescimento das formas promastigotas em menos de 7 horas (dados não mostrados), porém esse efeito foi relacionado provavelmente a uma atividade citotóxica inespecífica, uma vez que nessas mesmas concentrações também houve morte de $100 \%$ dos macrófagos.

Estes resultados nos permitem concluir que o extrato hidroalcoólico de folhas de $S$. cayennensis possui atividade inibitória tanto para L. amazonensis quanto para L. braziliensis e sugere futuros estudos com compostos isolados deste extrato, bem como a avaliação do seu efeito em modelos de infecção in vitro e in vivo.

\section{AGRADECIMENTOS}

Ao $\mathrm{CNPq}$ pelo auxílio financeiro (Proc. $n^{\circ}$ 620081/2004-0 ACT), pelas bolsas PIBIC para G.C.Costa, I.C.D.S.Carvalho e pela bolsa de Pós-doc de R.N.M.Guerra. À FAPEMA pela bolsa de mestrado de J.L. Bezerra. À Dra. Aldina Barral do Centro de Pesquisas Gonçalo Moniz- CPqGM-FIOCRUZ/BA, por fornecer as formas promastigotas de L. amazonensis e L. braziliensis utilizadas neste estudo. A Dra. Maria Jânia Teixeira do CPqGM-FIOCRUZ/BA, por colaborar na execução dos testes laboratoriais.

\section{REFERÊNCIAS}

Akendengue B, Ngou-milama E, Laurens A, Hocquemiller R 1999. Recent advances in the fight against leishmaniasis with natural products. Parasite 6: 3-8.

Araújo CAC, Alegrio LV, Leon LL 1998. Antileishmanial activity of compounds extracted and characterized from Centrolobium sclerophyllum. Phytochemistry 49: 751-754.

Barata LES, Santos, LS, Ferri PH, Phillipson JD, Paine A, Croft SL 2000. Anti-leishmanial activity of neolignans from Virola species and synthetic analogues. Phytochemistry 55: 589-595.

Bergmann BR, Costa SS, Moraes VLG 1997. Brazilian medicinal plants: a rich source of immunomodulatory substances. Braz J Assoc Adv Sci 49: 395-402.

Bezerra JL, Costa GC, Lopes TC, Carvalho ICDS, Patrício FJ, Sousa SM, Amaral FMM, Rebelo JMM, Guerra RNM, Ribeiro MNS, Nascimento FRF 2006. Avaliação da atividade leishmanicida in vitro de plantas medicinais. Rev Bras Farmacogn 16(Supl.): 631-637.

Camacho MR, Phillipson JD, Croft LS, Kirby GC, Warhurst DC, Solis PN 2000. Terpenoids from Guarea rhophalocarpa. Phytochemistry 56: 203-210.

Camacho MR, Phillipson SL, Croft PN, Marshall SJ, Ghazanfar SA 2003. Screening of plants extracts for antiprotozoal and cytotoxic activities. J Ethnopharmacol 89: 185191.

Caribe J, Campos JM. 1991. Plantas que ajudam o homem. Guia Prático para Época. Atual.

Chen M, Christensen SB, Blom J, Lemmich E, Nadelmann L, Fich K, Theander TG, Kharazmi A 1993. Licochalcone A, a novel antiparasitic agent with potent activity against human pathogenic protozoan species of Leishmania. Antimicrob Agents Chemoth 37: 25502556.

Costa JML, Vale KC, França F, Saldanha ACR, Silva JO, Lago EL, Marsden PD, Magalhães AV, Silva CMP, Netto AS, Galvão CES 1990. Cura espontânea da leishmaniose causada por Leishmania (Viannia) braziliensis em lesões cutâneas. Rev Soc Bras Med Trop 23: 205-208.

Croft SL, Yardley V 2002. Chemotherapy of leismaniasis. Curr 
Pharm Design 8: 319-342.

Duarte MCT, Figueira GM, Pereira B, Magalhães PM, Delarmelina C 2004. Atividade antimicrobina de extratos hidroalcoólicos de espécies da coleção de plantas medicinais CPQBA/UNICAMP. Rev Bras Farmacogn 14(Supl. 1): 6-8.

Falcão HS, Lima IO, Santos VL, Dantas HF, Diniz MFFM, Barbosa-Filho JM, Batista LM 2005. Review of the plants with anti-inflammatory activity studied in Brazil. Rev Bras Farmacogn 15: 381-391.

Fournet A, Barrios AA, Munõz V, Hocquemiller CA 1992a. Effects of natural naphtoquinones in Balb/c mice infected with Leishmania amazonensis and $L$. venezuelensis. Ann Trop Med Parasit 43: 219-222.

Fournet A, Angelo A, Muñoz V, Roblot F, Hocquemiller R, Cavé A 1992b. Biological and chemical studies of Pera benensis, a Bolivian plant used in folk medicine as a treatment of cutaneous leishmaniasis. $J$ Ethnopharmacol 37: 159-164.

Fournet A, Gantier JC, Gautheret L, Leysalles M H, Munos J, Mayrargues H, Moskowitz R, Cavé A 1994. The activity of 2-substituted quinoline alkaloids in Balb/c mice infected with Leishmania donovani.J Antimicrob Chemoth 33: 537-544.

Fournet A, Ferreira ME, Rojas de Arias A, Torres de Ortiz S, Fuentes S, Nakayama H, Schinini A 1996. In vitro efficacy of oral and intralesional administration of 2-substituted quinolines in experimental treatment of new world cutaneous leishmaniasis caused by Leishmania amazonensis. Antimicrob Agents Chemoth 40: 2447-2451.

França F, Cuba CA, Moreira EA, Almeida M, das Virgens ML, Marsden PD 1993. Avaliação do efeito do extrato de casca de cajueiro-branco (Anacardium occidentale) sobre a infecção por Leishmania (Viannia) braziliensis. Rev Soc Bras Med Trop 26: 151-155.

Iwu MM, Jackson JE, Schuster BG 1994. Medicinal plants in the fight against leishmaniasis. Parasitol Today 10: 65-68.

Kam TS, Sim KM, Koyana T, Toyoshima M, Hayash M, Komiyama K 1997. Citotoxic and Leishmanicidal aminoglycosteroids and aminosteroids from Holarrhena curtisii. J Nat Prod 61: 1332-1336.

Kayser O, Kiderlen AF, Laatsch H, Croft SL 2000. In vitro leishmanicidal activity of monomeric and dimeric naphthoquinones. Acta Tropica 77: 307-314.

Lopes SP 1977. Flora de Venezuela -Verbenaceae. Ed. Universidad de Los Andes, Venezuela..

Mahiou V, Roblot F, Hocquemiller R, Cave A, Rojas de Arias A, Inchausti A, Yaluff G, Fournet A 1994. Aporphine alkaloids from Guatteria foliosa. J Nat Prod 57: 890895.

Marsden PD, Tada MS, Barreto AC, Cuba CC 1984. Spontaneous healing of Leishmania braziliensis braziliensis skin ulcers. Trans Royal Soc Trop Med Hyg 78: 561-562.

Marsden PD 1994. Personal experience with diagnostic and therapeutic aspects of human Leismania (Viannia) braziliensis in Três Braços. Mem I Oswaldo Cruz 89: 485-487.

Matthias LA, Emily A 1993. Tapping and Amazonian plethora: four medicinal plants of Marajó Island, Pará-Brazil. $J$ Ethnopharmacol 40: 53-75.

Mesia-Vela S, Souccar C, Lima-Landman MT, Lapa AJ 2004.
Pharmacological study of Stachytarpheta cayennensis Vahl in rodents. Phytomedicine 11: 616-624.

Mittal N, Gupta N, Saksena S, Goyal N, Roy U, Rastogi AK 1998. Protective effect of picrolive from Pichrorhiza kurroa against Leishmania donovani infections in Mesocricetus auratus. Life Sci 63: 1823-1834.

Moreira RCR, Costa JML, Saldanha AC, Silva AR 1998. Projeto Buriticupu Maranhão II. Plantas usadas como terapêutica da leishmaniose tegumentar americana na região de Buriticupu-Maranhão. Rev Soc Bras Med Trop 31(Supl. I): T248, 126.

Moreira RCR, Rebêlo JMM, Gama MEA, Costa JML 2002. Nível de conhecimento sobre Leishmaniose Tegumentar Americana (LTA) e uso de terapias alternativas por populações de uma área endêmica da Amazônia do Maranhão, Brasil. Caderno de Saúde Pública 18: 187-195.

Mosmann T 1983. Rapid colorimetric assay for cellular growth and survival: application to proliferation and cytotoxicity assays. J Immunol Methods 16: 55-63.

Netto EM, Tada MS, Golight KDC, Lago E, Barreto AC, Marsden P 1985. Conceitos de uma população local a respeito da leishmaniose mucocutânea em uma área endêmica. Rev Soc Bras Med Trop 18: 33-37.

Olliaro PL, Bryceson ADM 1997. Pratical progress and new drugs for changing patterns of leishmaniasis. Parasitol Today 9: 323-328.

Paula-Junior W, Rocha FH, Donatti L, Fadel-Picheth CMT, Weffort-Santos AM 2006. Leishmanicidal, antibacterial, and antioxidant activities of Caryocar brasiliense Cambess leaves hydroethanolic extract. Rev Bras Farmacogn 16(Supl.): 625-630.

Phillipson JD, Wright CW 1991a. Medicinal plants in tropical medicine: Medicinal plants against protozoal diseases. Trans Royal Soc Trop Med Hyg 85: 18-21.

Phillipson JD, Wright CW 1991b. Antiprotozoal agents from plant sources. Planta Med 57: 53-59.

Pio Corrêa P 1984. Dicionário das plantas úteis do Brasil e das exóticas cultivadas. vol. 5. Imprensa Nacional, Rio de Janeiro, Ministério da Agricultura, Rio de Janeiro, Brasil.

Queiroz EF, Roblot F, Cave A, Paulo MD, Fournet A 1996. Pessoine and spinosine, two catecholic berbines from Annona spinescens. J Nat Prod 59: 438-440.

Rossel RA, Duran RJ, Rossel O, Rodrigues AM 1992. Is leishmaniasis ever cured? Trans Royal Soc Trop Med Hyg 86: 251-253.

Schapoval EES, Winter DE, Vargas MR, Chaves CG, Raquel BJA, Zuanazzi ATH 1998. Antiinflammatory and antinoceptive activities of extracts and isolated compounds from Stachytarpheta cayennensis. J Ethnopharmacol 60: 53-59.

Schmeda-Hirschmann G, Razmilic I, Sauvain M, Moretti C, Munoz V 1996. Antiprotozoal activity of jatrogrossidione from Jatropha grossidentada and jatrophone from Jatropha isabelli. Phytother Res 10: 375-378.

Troncoso, NS 1979. Verbenaceae. In: Burkart, A. (Org.) Flora Ilustrada de Entre Rios, vol. 6., pt. 5. INTA, Buenos Aires, pp. 229-294.

Yang DM, Liew FY 1992. Effects of quinghaosu (artemisin) and its derivates on experimental cutaneos leismaniasis. Parasitology 106: 7-11. 